\title{
REVIEWER LIST
}

\section{The journal would like to recognize the following individuals who have reviewed manuscripts during the past year. We thank you for your continued support.}

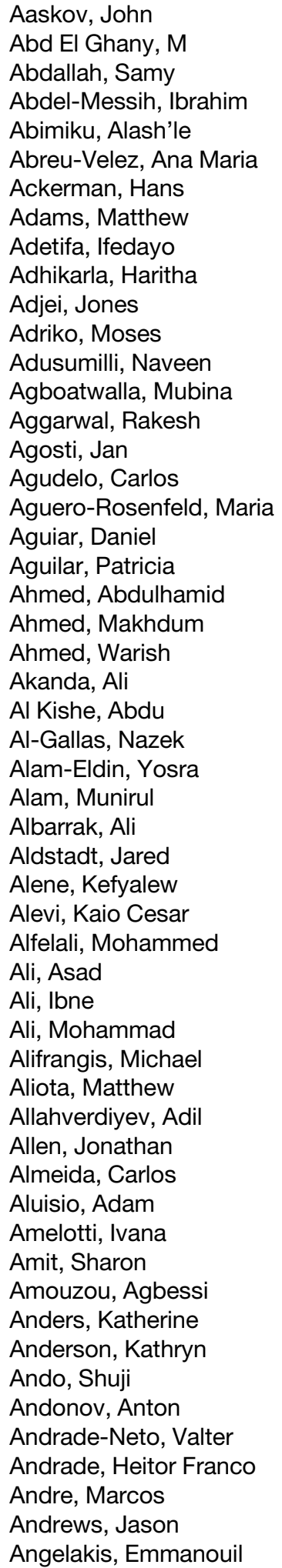

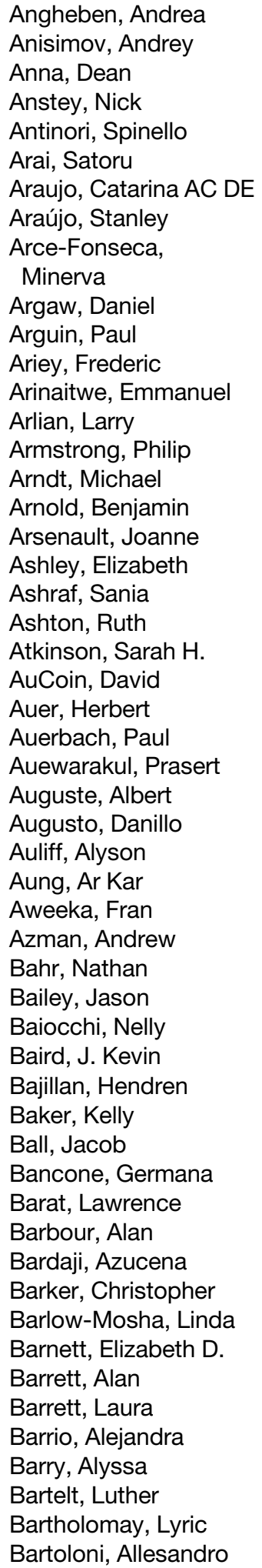

Bassat, Quique
Bassiri-Jahromi,
Shahindokht
Battle, Katherine
Batty, Kevin
Bausch, Daniel
Beasley, David
Becker, Norbert
Bello, Salvador
Benard, Gil
Benazouz, Mustapha
Bendall, Justin
Benedict, Mark
Bennett, Adam
Bennuru, Sasisekhar
Berendes, David
Berg, Douglas
Bergeron, Eric
Berman, Jonathan
Bern, Caryn
Bernard, Kristen
Bernigaud, Charlotte
Berry, Thomas D.
Beshir, Khalid
Besim, Hasan
Beverley, Stephen
Bhattarai, Achuyt
Billingsley, Peter
Birbeck, Gretchen
Bird, Brian
Biritwum, Nana Kwadwo
Birnie, Emma
Bisanzio, Donal
Bisoffi, Zeno
Black IV, William
Blacksell, Stuart
Blair, Carol
Blair, Patrick
Blaney, David
Blanton, Lucas
Blau, Dianna
Blitvich, Bradley
Blum, Johannes
Boakye, Daniel
Boehm, Alexandria
Boelaert, Marleen
Boeras, Debi
Boggild, Andrea
Bolling, Bethany
Bonifaz, Alexandro
Bonnet, Maryline
Borchers, Christoph
Borges, Vitor
Bosco-Lauth, Angela

Bosman, Andrea Bothamley, Graham Bottieau, Emmanuel Boum II, Yap Bousema, Teun Boussaa, Samia Boussinesq, Michel Bowen, Richard Bower, Hilary Bowman, Dwight Bowman, Natalie Bradley, Kristy Brady, Oliver Braslins, Philip Brault, Aaron Bravo, Francisco Brehm, Klaus Breman, Joel Brennan, Benjamin Brennan, Patrick Brewer, Matthew Briand, Valerie Brickley, Elizabeth Brito, Cristiana Brittingham, Andrew Broadhurst, Jana Brodskyn, Claudia Brooks, Yolanda Brouwer, Kimberly Brown, James Brown, Joe brunetti, enrico Bruschi, Fabrizio Buccheri, Renata Buckner, Frederick Budge, Philip Budke, Christine Buekens, Pierre Buhagiar, Tamara Bui, David Bumb, Ram Burgos, Juan Manuel Burke, Rachel Burkett-Cadena, Nathan Burkovski, Andreas Burton, Matthew Busch, Maria Busch, Michael Bustos, Javier Butler, Thomas Cabada, Miguel Cabié, André Calisher, Charles Calvert, Amanda 


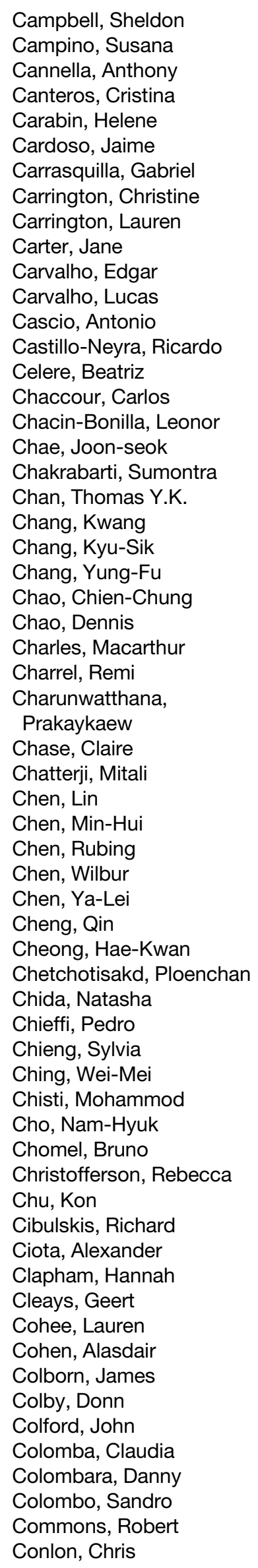

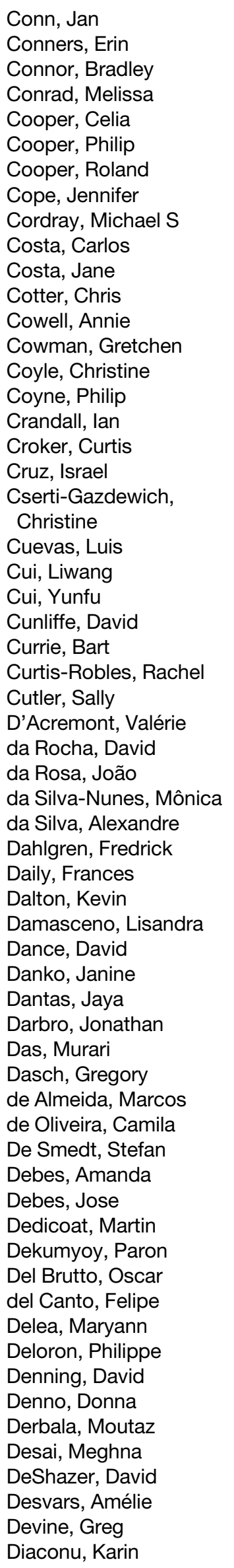

\begin{tabular}{|c|c|}
\hline Diallo, Karidia & Felger, Ingrid \\
\hline Diaz, James & Feng, Jun \\
\hline Dickinson, Katherine & Ferguson, Mike \\
\hline Diez, Cristina & Fernandez, Stefan \\
\hline Ding, Xavier & Ferreira, Marcelo \\
\hline Dittrich, Sabine & Fierer, Joshua \\
\hline Divala, Titus & Fiore, Anthony \\
\hline Dobbs, Katherine & Fischer, Marc \\
\hline Doherty, Anne & Fischer, Peter \\
\hline Domingo, Gonzalo & Fleury, Agnes \\
\hline Dorlo, Thomas & Florin, David \\
\hline Dorn, Patricia & Flueckiger, Rebecca \\
\hline Douglass, Jan & Fluegge, Kyle \\
\hline Drakeley, Chris & Foley, Janet \\
\hline Drebot, Mike & Fölster-Holst, R \\
\hline Dreibelbis, Robert & Fonseca, Ana Gloria \\
\hline Drexler, Naomi & Fooks, Anthony \\
\hline Driscoll, Amanda & Foradori, Matthew \\
\hline Dubey, JP & Forrester, Janet \\
\hline Dubey, Vikash & Forshey, Brett \\
\hline Duggal, Nisha & Fournier, Pierre \\
\hline Duggal, Nisha & Fox, Jay W. \\
\hline Duggan, Christopher & Foy, Brian \\
\hline Dunn, Julia & Frangoulidis, Dimitrios \\
\hline Dupnik, Kathryn & Frean, John \\
\hline DuPont, Herbert & Freedman, David \\
\hline Dupuis, Alan & Freeman, Matthew \\
\hline Duron, Olivier & Friedman, Jennifer \\
\hline Duthie, Malcolm & Friedman, Melissa \\
\hline Dutta, Prafulla & Fritsche, Thomas \\
\hline Dvorak, Jan & Fronteira, Ines \\
\hline Ebel, Gregory & Fumadó, Victoria \\
\hline Eberhard, Mark & Furin, Jennifer \\
\hline Ebi, Kristie & Gaff, Holly \\
\hline Eckerle, Michelle & Galappaththy, Gawrie \\
\hline Eckhoff, Philip & Galdos-Cardenas, Gerson \\
\hline Edwards, Morven & Galel, Susan \\
\hline Efstratiou, Androulla & Gamboa, Dionicia \\
\hline Eibach, Daniel & Gárate, Teresa \\
\hline Elafros, Melissa & Garbino, José \\
\hline Elliott, Ivo & Garcia, Hector \\
\hline Ellis, Michael & Garcia, Lynne \\
\hline Emeto, Theophilus & Garcia, Melissa \\
\hline Endy, Timothy & Gardenal, Cristina \\
\hline Engelman, Daniel & Garfunkel, Lynn \\
\hline Engleberg, Niels & Garn, Joshua \\
\hline English, Mike & Garry, Robert \\
\hline Epstein, Judith & Gass, Katie \\
\hline Ercumen, Ayse & Gautam, Om \\
\hline Errea, Renato & Gavidia, Cesar \\
\hline Escalante, Ananias & Gawoski, John \\
\hline Estévez, Alejandra & Geary, Timothy \\
\hline Ettestad, Paul & Gerez de Burgos, Nelia \\
\hline Eugenin, Eliseo & Ghildyal, Reena \\
\hline Evans, Carlton & Ghrab, Jamila \\
\hline Evans, Darin & Gies, Sabine \\
\hline Failoc-Rojas, Virgilio & Gil, Ana \\
\hline Fairhurst, Rick & Gimnig, John \\
\hline Fairley, Jessica & Gladding, Sophia \\
\hline Famrakiotis, Dimitrios & Goarant, Cyrille \\
\hline Fan, CK & Godsey, Marvin \\
\hline Feldmeier, Hermann & Goeijenbier, Marco \\
\hline
\end{tabular}


Gold, Howard

Goldfarb, David

Gollob, Kenneth

Gomes-Solecki, Maria

Gomes, Vieira Coutinho

Abreu

Gomez Barrio, Alicia

Gomez Marin, Jorge

Gonzalez, Angel

González, Daniel

Goris, Marga

Gosling, Roly

Goswami, Neela

Gottschalk, Marcelo

Gould, Carolyn

Govender, Nelesh

Graff, Joel

Graham-Brown, John

Graves, Stephen

Green-Hines, Andrea

Green, Micheal

Greenhouse, Bryan

Gregory, Christopher

Griekspoor, Andre

Grigg, Matthew

Grobusch, Martin

Gruber, Joann

Guerra Gronerth, Rosio

Guerra, Ana

Guerra, Humberto

Guerrant, Richard

Guerrero-Wooley, Richelle

Guerry, Patricia

Guest, Bailey

Gundacker, Nathan

Gupte, Akshay

Gupte, Mohan

gurley, emily

Gurtler, Ricardo

Gute, David

Gutierrez, Ramiro

Gutman, Julie

Guzman, Manuel

Haddow, Andrew

Hahn, William

Hajjou, Mustapha

Hakami, Ramin

Hall, Eric

Hallegraeff, Gustaaf

Halsey, Eric

Halstead, Scott

Hamer, Davidson

Hamer, Gabriel

Hanley, Kathryn

Hanrahan, Colleen

Harms, Gundel

Harvey, Steven

Hasker, Epco

Hatakeyama, Shuji

Hawkes, Michael

Hegazy, yamen

Heiden, David
Heitzinger, Kristen

Held, Jana

Hendel-Paterson, Brett

Henning, Lars

Heo, Sang Taek

Herbreteau, Vincent

Herrera, Sócrates

Herrick, Jesica

Herrington, James

Hetzel, Manuel

Hickey, Patrick

Hidalgo, Marylin

Hifumi, Toru

Hills, Susan

Hirai, Mitsuaki

Hisada, Michie

Hobson-Peters, Jody

Hochberg, Natasha

Holbrook, Mike

Holland, Martin

Holzman, Samuel

Hombach, Joachim

Honein, Margaret

Hong, Sung-Tae

Hooli, Shubhada

Hopkins, Heidi

Horowitz, Harold

Horstick, Olaf

Hospenthal, Duane

Hossain, Mohammad

Houpt, Eric

Hovius, Joppe

Howard, Guy

Howes, Rosalind

Howlett, William

Huang, Claire

Huang, Claire

Huddart, Sophie

Huebner, Marc

Huits, Ralph

Humphrey, Jean

Hung, Yao-Min

Hunsperger, Elizabeth

Hynes, Noreen

lannaccone, Marco

Ikegami, Tetsuro

Ikpatt, Offiong Francis

Inauen, Dr. Jennifer

Intapan, Pewpan

Ippolito, Matthew

Irish, Seth

Irving, Wiliam

Ismail, Ahmad

Izopet, Jacques

Izurieta, Ricardo

Jackson, Alan

Jackson, Ronald

Jackson, Yves

Jacob, Shevin

Jacobson, Karen

Jain, Vivek

Jänisch, Thomas
Janjua, Naveed

Jeandron, Aurelie

Jeelani, Suhail

Jeronimo, Selma

Jha, T.K.

Johansson, Michael

John, Chandy

Johnson, Barbara

Johnson, Paul

Johnson, Ray

Jones, Malcolm

Junglen, Sandra

Kaba, Mamadou

Kabaalioglu, Adnan

Kalayanarooj, Siripen

kalita, jayantee

Källander, Karin

Kallio, Eva R.

Kamau, Edwin

Kaminstein, Daniel

Kang, Ki-Woon

Kansiime, Catherine

Kapur, Anil

Karanis, Panagiotis

Kariuki, Sam

Karl, Stephan

Kaur, Harparkash

Kawabata, Hiroki

Keating, Joseph

Keiser, Jennifer

Kelly, Paul

Keness, Yoram

Kenney, Joan

Kersh, Gil

Ketheesan, Natkunam

Ketzis, Jennifer

Keystone, Jay

Khan, Wasif

Khush, Ranjiv

Kidd, Sarah

Kilgore, Paul

Killeen, Gerry

Kim, Dong-Min

King, Charles

King, Christopher

Kirby, Amy

Kirchhoff, Louis

Kirenga, Bruce

Kitua, Andrew

Kjetland, Eyrun

Klein, William

Klion, Amy

Knowlton, Kim

Kochel, Tadeusz

Kocken, Clemens

Koenig, Serena

Kolars, Joseph

Komar, Nicholas

Koporc, Kim

Kosoy, Michael

Kozubsky, Leonora

Krause, Peter
Kremsner, Peter

Kroupin, Georgi

Kuhn, Walter

Kuilman, Luppo

Kumar, Nirbhay

Kumpel, Emily

Kunin, Calvin

Kurth, Florian

Kutsuna, Satoshi

Kvalsvig, Jane

Kyle, Dennis

LaBeaud, Angelle Desiree

Lachish, Tamar

Lages-Silva, Eliane

Lammie, Patrick

Landis, Clive

Lang, Dennis

Langelier, Charles

Langley, Rick

Lantagne, Daniele

Laporta, Gabriel

Larkins, Sarah

Larson, Bruce

Larson, Leila

Laurens, Matthew

Lavery, Jim

Lawler, James

Lawniczak, Mara

Lazzari, Claudio

Lee, Chang-Seop

Lee, Keun Hwa

Lehmann, Tovi

Lemos, Elba

Lenin, Luis

Leontsini, Elli

Lerdthusnee, Kriangkrai

Letson, George

Levett, Paul

Lewis, Richard

Lewis, Sheri

$\mathrm{Li}$, Shizhu

$\mathrm{Li}, \mathrm{Xi}$

Liautaud, Bernard

Liebman, Kelly

Lietman, Thomas

Lightowlers, Marshal

Lillebaek, Troels

Limmathurotsakul, Direk

Lin, Audrie

Lin, Jessica

Lin, Yung-Ching

Lindberg, Lene

Linder, Ewert

Lindo, John

Lindsay, Robbin

Lindsay, Steve

Llanos Cuentas, Alejandro

Lloyd, Linda

Lobo, Neil

Londono-Renteria, Berlin

Lopez-Verges, Sandra

Lopez, Velma 


\author{
Lord, Cynthia \\ Lorenzo-Morales, Jacob \\ Lötsch, Felix \\ Lucchini, Anna \\ Luo, Tao \\ Lushbaugh, William \\ Lustigman, Sara \\ Luz, Kleber \\ Mabey, David \\ MacDonald, Margaret \\ Machado, Gustavo \\ Machado, Paulo \\ Machado, Ricardo \\ Maciver, Sutherland \\ Mackenzie, Charles \\ Macnaghten, Philip \\ MacNeil, Adam \\ Madan, Rajat \\ MAertzdorf, Joroen \\ Magnusssen, Pascal \\ Maguire, Jason \\ Mahajan, Vikram \\ Maharaj, Payal \\ Mahdy, Mohammed \\ Malavige, Neelika \\ Maleewong, Wanchai \\ Malik, Richard \\ Malone, Robert \\ Maloney, Samuel \\ Marcos, Luis \\ Markle, William \\ Marks, Michael \\ Marquis, Grace \\ Marrie, Thomas \\ Martin, Stacey \\ Martinez Ibarra, Jose \\ Martinez-Giron, Rafael \\ Martinez, Eric \\ Marx, Preston \\ Mason, Carl \\ Masur, Henry \\ Mathema, Barun \\ Mathur, Vijay \\ Matlashewski, Greg \\ Matsunaga, Jim \\ Maurin, Max \\ Maves, Ryan \\ May, Fiona \\ Mayxay, Mayfong \\ Mbacham, Wilfred \\ Mbogo, Charles \\ Mbulaiteye, Sam \\ McArthur, Monica \\ McAteer, Jarred \\ McBain-Rigg, Kristin \\ McBride, Jere \\ McBride, John \\ McBryde, Emma \\ McCord, Gordon \\ McDonald, Emily \\ McDonald, Erin \\ McGwire, Bradford
}

McKenna, Megan

McMahon-Pratt, Diane

McManus, Donald

McQuiston, Jennifer

Mduluza, Takafira

Meagher, Robert

Mediannikov, Oleg

Mehta, Sanjay

Mehtar, Shaheen

Melby, Peter

Meltzer, Eyal

Mendez, Diana

Mendez, Jairo

Merker, Matthias

Meshnick, Steve

Messenger, Louisa

Mestra, Laureano

Meyer, Sarah

Meymandi, Sheba

Michelow, Ian

Mihalca, Andrei Daniel

Mikoleit, Matthew

Mileno, Maria

Miller, $\mathrm{F}$.

Miller, Nathan

Milligan, Paul

Mills, Harriet

Milner, Kate

Mitre, Edward

Miyamura, Tatsuo

Mohanty, Sanjib

Mokuolu, Olugbenga

Molina, Israel

Mondal, Dinesh

Monge-Maillo, Begoña

Montalvo, Ana-Margarita

Montgomery, Susan

Montresor, Antonio

Moore, Cindy

Moraes, Luiz Roberto

Morawski, Bozena

Mordmueller, Benjamin

Morita, Masatomo

Moro, Pedro

Mosler, Hans-Joachim

Moss, William

Moss, William

Mossel, Eric

Moure, Zaira

Moyo, Sabrina

Mu, Jianbing

Mueller, Judith

Muga, Geoffrey

Mukhtar, Maowia

Müller, Olaf

Mumcuoglu, Kosta

Murray, Kristy

Murray, Megan

Muzari, Odwell

Nacher, Mathieu

Naquira, Cesar

Nascimento, Erika
Nash, Scott

Nash, Theodore

Nayyar, Gaurvika

Ncayiyana, Jubilani

Nelson, Kara

Nelson, Kenrad

Nemeth, Nicole

Neto, Daniel

Neumayr, Andreas

Nevin, Remington

Newton, Paul

Ngure, Francis

Nickerson, Emma

Nielsen, Morten

Nierenberg, Natalie

Nilles, Eric

Nkouawa, Agathe

Nogueira, Maurício

Nolan, Thomas

Noordin, Rahmah

Norris, Douglas

Norton, Robert

Novaes Ramos, Alberto

Nsobya, Samuel

Null, Clair

Nusalak, Ananda

Nutman, Thomas

O'Connell, Elise

Oberhelman, Richard

Ogbuagu, Onyema

Ohnishi, Kenji

Okell, Lucy

Olayinka, Adebola

Oleson, Jacob

Oliva, Gaetano

Oliveira, Everton

Oliveira, Fabiano

Olson, Daniel

Omeara, Wendy

Omotayo, Moshood

Ooi, Eng Eong

Oren, Eyal

Oriero, Eniyou

Osoro, Eric

Ostera, Graciela

Oteo, José Antonio

Otranto, Domenico

Owers, Katharine

Paddock, Christopher

Padgett, Kerry

Palekar, Rakhee

Palma, Gloria

Pandey, Deb

Panzera, Francisco

Paploski, Igor

Parikh, Sunil

Paris, Daniel

Pasqualotto, Alessandro

Pathmanathan, Ishani

Paul, Jean

Pavlinac, Patricia
Pearson, Richard

Pearson, Talima

Pedersen, Kathy

Peeters Grietens, Koen

Peng, Hong-Juan

Perera, Rushika

Perez-Gracia, Maria Teresa

Perez, Ana Rosa

Perkins, Alex

Peter, Dincy

Peters, Remco

Petersen, Christine

Petersen, Eskild

Petersen, Lyle

Petri, William

Petruccelli, Bruno

Pham, Bang

Piarroux, Renaud

Picardeau, Mathieu

Pickering, Amy

Pierce, Kristen

Pietrantonio, Patricia

Pinto, Ana Yecê

Pita, Daniela

Pita, Sebastian

Poespoprodjo, Jeanne

Ponzio, Todd

Porter, Chad

Postels, Douglas

Povlich, Laura

Powers, Ann

Prakash, John

Prendergast, Andrew

Price, Erin

Price, Ric

Pritt, Bobbi

Prue, Christine

Putnam, Shannon

Puttkammer, Nancy

Quinnell, Rupert

Quintanilla-Licea, Ramiro

Raabe, Vanessa

Rabinovich, Regina

Radke, Elizabeth

Raghavan, Ram

Raguin, Gilles

Rahman, Kazi

Rahman, Kazi

Ralph, Anna

Ramamurthy,

Thandavarayan

Ramful, Duksha

Ramírez Soto, Max Carlos

Ranque, Stéphane

Rapaka, Rekha

Ray, Isha

Read, Jennifer

Reed, Brian

Reed, Steven

Reid, Stewart

Reidl, Julia

Reis-Santos, Barbara 
Reisen, William

Reithinger, Richard

Reller, Megan

Requena-Méndez, Ana

Reves, Randall

Reyburn, Hugh

RG de Freitas, Marcos

Ribeiro, Aline

Ricaldi, Jessica

Richards, Jack

Riddle, Mark

Rieder, Hans

Rigatto, Maria Helena

Riley, Lee

Ringwald, Pascal

Rivera, Windell

Rivers, Caitlin

Roberts, Tamalee

Roberts, Teri

Robertson, Gemma

Robertson, Jane

Robinson, Leanne

Robinson, Matthew

Rockabrand, David

Rodriguez-Morales,

Alfonso

Rodriguez-Morales,

Alfonso J.

Rodriguez-Perez,

Mario

Rodriguez, Isabel

Roestenberg, Meta

Rogawski, Elizabeth

Roldán, William

Root, Jeff

Roque, André Luiz

Rosen, Jennifer

Rosenthal, Philip

Rossi, Shannan

Roth, Lukas

Rothman, Alan

Rowe, Alexander

Rubach, Matthew

Rubio, Jose

Rueckert, Claudia

Rugemalila, Joas

Rugwizangoga, Belson

Rungta, Narendra

Rupprecht, Charles

Ryoo, Sungweon

Saarma, Urmas

Sacks, David

Saito, Tais

Sakanari, Judy

Salas-Coronas, Joaquin

Sales, Carolina

Salje, Henrik

Salvador, Fernando

San Martin, Juan Victor

Sanchez, Ana

Sanchez, Jose

Sanders, John
Santiago, Gilberto

Santos, Ana Sofia

Sato, Marcello

Sato, Shingo

Satoskar, Abhay

Saylors, Karen

Sbrana, Elena

Scarpino, Samuel

Schieffelin, John

Schilling, Katharine

Schmidt, Wolf-Peter

Schoepp, Randal

Schriefer, Albert

Schully, Kevin

Schulz, Thomas

Schwab, Kellog

Schwartz, Eli

Schwartzman,

Joseph

Scorpio, Diana

Scott, Alan

Searle, Kelly

Seas, Carlos

Secor, William

Sejvar, James

Sekeyova, Zuzana

Seligman, Stephen

Sexton, Daniel

Seydel, Karl

Shadomy, Sean

Sharp, Tyler

Shelly, Mark

Shepard, Donald

Shetty, Avinash

Shimasaki, Teppei

Shirley, Debbie-Ann

Shlim, David

Shott, Joseph

Shrestha, Kasturi

Shretta, Rima

Shubert, Chuck

Sibley, Carol

Silberberg, Donald

Simons, Mark

Singer, Steven

Singh, SP

Sinharoy, Sheela

Siqueira-Neto, Jair

Siqueira, Andre

Siqueira, Isadora

Slapeta, Jan

Slesak, Günther

Slutsker, Laurence

Smith, David

Smith, James

Smith, Tom

Snow, Bob

Snowden, Karen

Soares, Neci

Solomon, Anthony

Sondorp, Egbert

Soong, Lynn
Soto, Jaime

Sousa, Fernanda

Souza, Marcy

Spellberg, Brad

Spiers, Angus

Spotin, Adel

Stallknecht, David

Stauber, Christine

Stefanoff, Pawel

Steffen, Robert

Steinmann, Peter

Steketee, Richard

Stenos, John

Stephens, Dianne

Stigler Granados, Paula

Stijnis, Cees

Stillwaggon, Eileen

Stockman, Douglas

Stojkovic, Marija

Stone, William

Stresman, Gillian

Stroppa, María

Sultana, Rebeca

Summers, Andrea

Suzuki, Yasuhiro

Swanson, Robert

Tabernero Estevez,

Patricia

Takala-Harrison,

Shannon

Takhampunya, Ratree

Takiff, Howard

Tamarozzi, Francesca

Tan, Christina

Tanner, Marcel

Tapia, Milagritos

Tarr, Phillip

Tatem, Andrew

Tauxe, Robert

Taylor, allan

Taylor, Martin

Taylor, Steve

Taylor, Terrie

Taylor, Walter

Telford, Sam

Tesana, Smarn

Tesfahun, Esubalew

Tesh, Robert

Tetteh, Kevin

Tewari, Nitesh

Thevos, Angelica

Thiele, Elizabeth

Thipmontree, Wilawan

Thomas, Evan

Tiberi, Simon

Tisch, Daniel

Tobon, Angela

Toledo, Rafael

Topp, Stephanie

Tornheim, Jeffrey

Toro, Carlos

Trumbo, Craig
Tuft, Stephen

Turner, Paul

Turner, Richard

Tusting, Lucy

Tyler, Kenneth

Udhyakumar, V.

Uehara, Yuki

Ulrich, Rainer

Uneke, Chigozie

Unnasch, Thomas

Unnikrishnan, Avinash

Vairo, Francesco

Valero, M. Adela

Valete-Rosalino, Claudia

van den Hurk, Andrew

van der Hoek, Wim

van Eijk, Anna Maria

van Griensven, Johan

Vanlandingham, Dana

Vasilakis, Nikolaos

Vazquez, Gonzalo

Venegas, Juan

Vercruysse, Jozef

Verghese, Valsan

Vermund, Sten

Verweij, Jaco

Vignier, Nicolas

Vikrant, Sanjay

Villanueva, Sharon Yvette

Angelina

Vincent, Veronique

Viney, Kerri

Vinikoor, Michael

Visser, Benjamin

Viswanathan, Stalin

Viveiros, Miguel

von Seidlein, Lorenz

Vu, David

Waggoner, Jesse

Wagner, Dave

Walker, Billy

Walker, David

Walsh, Douglas

Walson, Judd

Wang Di, Kinley

Wang, Tian

Wareth, Gamal

Warrell, David

Wasley, Annemarie

Wassmer, Samuel

Waters, Norman

Watt, George

Watts, Douglas

Watts, Matthew

Weatherhead, Jill

Weaver, Scott

Weber, Stefan

Wegmüller, Rita

Weil, Ana

Weinberger, $M$

Weiss, Louis

Wellinghausen, Nele 


\author{
Werneck, Guilherme \\ White, A. Clinton \\ White, Lisa \\ White, Michael \\ Whittaker, Maxine \\ Wickramasinghe, Susiji \\ Wieringa, Frank \\ Wijegunawardana, Asha \\ Wilby, Kyle John \\ Wilder-Smith, Annelies \\ Wilkins, Patricia \\ Wilkinson, Robert \\ Williams, Carolyn \\ Williamson, John
}

\author{
Wilson, Mark \\ Wilson, Mary \\ Wilson, Shona \\ Wilson, William \\ Winch, Peter \\ Winston, Alan \\ Winzeler, Elizabeth \\ Wirth, James \\ Wodecka, Beata \\ Wolf, Katherine \\ Wormser, Gary \\ Worrell, Caitlin \\ Wright, James \\ Wright, Richard
}

\author{
Wu, Zhongdao \\ Wunder, Elsio \\ Wuthiekanun, Vanaporn \\ Xu, Yihua \\ Yamasaki, Hiroshi \\ Yanagi, Hidetaka \\ Yansaneh, Aisha \\ Yates, John \\ Yolken, Robert \\ Yoon, In-Kyu \\ Yoshimura, Yukihiro \\ You, Danzhne \\ Young, Ed \\ young, Edward
}

\author{
Yu, Xuejie \\ Yukich, Joshua \\ Zaman, K \\ Zaman, Sayed \\ Zerpa Larrauri, Rito \\ Zhang, Furen \\ Zhang, Jianquan \\ Zhang, Jindong \\ Zhang, WenYi \\ Zhang, Xingyu \\ Zhu, Guan \\ Zimmerman, Peter \\ Zinszer, Kate \\ Zunt, Joseph
}

